\title{
Registering CIHR-funded randomized controlled trials: a global public good
}

\section{David Moher, Alan Bernstein}

See related article page 735

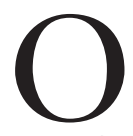
n July 26, 2004, the Canadian Institutes of Health Research (CIHR), the largest publicly funded granting agency in Canada, announced that all new CIHR-funded randomized controlled trials (RCTs) must be registered with an International Standard Randomised Controlled Trial Number (ISRCTN). ${ }^{1}$ Basic information about each registered trial will be posted on the public Web site of the ISRCTN register (www.controlledtrials.com), and the ISRCTN will be cited in any subsequent publication of trial results.

CIHR's decision to adopt an open trial-registry approach was based in large part on the set of values articulated in the Institutes' strategic plan, Blueprint 2007. ${ }^{2}$ Those values include the public interest, sound ethical principles, excellence, public transparency, accountability and collaboration. CIHR hopes that, among other benefits, trial registration will encourage and increase collaboration among researchers, the private sector and the community, reduce the risk of publication bias, reduce the wasteful duplication of research efforts, and contribute to global efforts to reduce or eliminate disease.

The call for trial registration is not new. ${ }^{3-5}$ Trial reporting standards, such as the CONSORT Statement, have strongly encouraged the use of a trial registration numbering system such as the ISRCTN, ${ }^{6}$ and the member journals of the International Committee of Medical Journals Editors will no longer consider unregistered RCTs for publication.?

Similarly, trial registries are not new; there are many in disparate forms and various clinical areas. ${ }^{8}$ CIHR's decision will cover the complete spectrum of trials, that is, those on pharmaceuticals, devices and surgical procedures as well as those examining psychosocial and health system interventions. Among the registration schemes available, CIHR has elected to use the ISRCTN. Increasingly, trials are conducted across geographic boundaries, making the ISRCTN a sensible choice, and a genuinely international one. Clearly, it would be desirable to have a single worldwide public database for all RCTs, regardless of funder and country. The ISRCTN was developed by the UK-based Current Controlled Trials, Ltd. (http://sciencenow.com/), a private Web-based publishing company that is supportive of transparency in clinical trials reporting. Each trial can be registered for Cdn\$120, a one-time fee to cover the costs of hosting, indexing and permanently displaying the trial record in the ISRCTN register. CIHR will cover the cost of registration for CIHR-funded trials. The registration fee can be waived at the discretion of the company for trials originating in low-income countries.

What are the benefits of trial registration? In a landmark study published nearly 20 years ago, Simes ${ }^{9}$ examined data contained in an oncology clinical trials registry and reported that statistically pooling the results of published trials only, compared with pooling published plus registered trials provided clinicians and patients alike with differing and opposing estimates of the effectiveness of a cancer intervention. Researchers are known to be more energetic about writing up reports of RCTs when the results are statistically positive. ${ }^{10}$ Likewise, journals seem more interested in publishing statistically positive reports. ${ }^{11}$ Such behaviours result in publication bias, as first reported more than 40 years ago. ${ }^{12}$ Although trial registration will not eliminate publication bias, for publicly funded trials it is likely to provide a degree of transparency and accountability not previously seen.

Even when the results of an RCT are statistically positive, some investigators attempt to report this information multiple times and in multiple ways, such as by publishing separately the results of each participating country or geographic region. Duplicate publication gives the unwary reader the impression of a far larger body of evidence pertaining to an intervention than actually exists. ${ }^{13}$ Such acts of duplication are likely to be reduced by the introduction of trial registration.

Knowledge of a trial's existence is particularly important to those who conduct systematic reviews. Such reviews are a fundamental building-block in the delivery of evidencebased health care. The results of systematic reviews will more likely produce biased results if they are missing information about the existence of trials and if covert duplicate publication results in double counting. Suboptimal systematic reviews are of little help to clinicians, clinical practice guideline developers, consumers and anyone else who uses them to inform decision-making.

Ultimately, trial registration will succeed only when all funders of randomized trials, including the pharmaceutical industry, come onboard. Pharmaceutical companies fund about $90 \%$ of RCTs performed globally. The decisions by GlaxoSmithKline and Eli Lilly to move toward trial registration are steps in the right direction.

Trial registration might also be the impetus for other good clinical trials practice. Chan and colleagues com- 
pared the contents of 102 trial protocols approved by the scientific ethics committees for Copenhagen and Frederiksberg, Denmark, during 1994 and 1995 with 122 subsequent publications. ${ }^{14}$ They reported that in nearly two thirds of the trials there was a change in at least one primary outcome between the protocol and publication. They also reported that statistically significant outcomes had a higher likelihood of being reported compared with nonsignificant outcomes. Similar results are reported in this issue of $C M A J .{ }^{15}$ Taken together, these results suggest that linking the ISRCTN with a publicly available protocol will be another positive step.

The business of RCTs is very large. CenterWatch, a Boston-based publishing and information services company focusing on the clinical trials industry, estimates that at least 41000 RCTs are currently in progress in North America (www.centerwatch.com).

Trial registration is an important initiative, but it is not a panacea. It will not provide access to trials submitted to regulatory agencies, a major source of trials. ${ }^{16}$ It will not in itself be sufficient to stop publication bias, duplicate publication or a plethora of questionable behaviours.

RCTs are central to the development of evidence-based health care. If we are to accelerate the development of costeffective new interventions, then open and public access to all trials and their outcomes will be key to achieving that goal. Transparency and accountability are important values in the work of the CIHR, and nowhere is that more visible and crucial than in research in which the immediate goal is to assess the safety and efficacy of a new drug, device, procedure or other intervention.

David Moher is from the Department of Pediatrics, Children's Hospital of Eastern Ontario Research Institute, and the Faculty of Medicine, University of Ottawa, Ottawa, Ont. Alan Bernstein is President of the Canadian Institutes of Health Research, Ottawa, Ont.

\section{Competing interests: None declared.}

Contributors: Dr. Moher conceived of the commentary. Drs. Moher and Bernstein participated in drafting and revising the article, and both approved the final version submitted for publication.
Acknowledgements: We thank Dr. Roderick McInnis, Isabelle Schmid and Terry Campbell, Canadian Institutes of Health Research; Dr. Douglas G. Altman, Cancer Research UK Medical Statistics Group; Dr. Gerd Antes, Director, German Cochrane Centre; and Dr. David L. Sackett, Trout Research and Education Centre at Irish Lake, Ont., for their helpful comments during the preparation of this manuscript.

\section{References}

1. Canadian Institutes of Health Research. CIHR joins in the international registration of randomised controlled trials [news release]. Available: www.cihrirsc.gc.ca/e/news/24107.shtml (accessed 2004 Aug 29).

2. Canadian Institutes of Health Research. Investing in Canada's future: CHIR's blueprint for health research and innovation. Available: www.cihrirsc.gc.ca/e/20266.html (accessed 2004 Aug 29).

3. Chalmers I, Dickersin K, Chalmers TC. Getting to grips with Archie Cochrane's agenda: all randomised controlled trials should be registered and reported. BM7 1992;305:786-7.

4. Dickersin K, Rennie D. Registering clinical trials. JAMA 2003;290:516-23.

5. Moher D. Clinical-trial registration: a call for its implementation in Canada. CMA7 1993;149:1657-8.

6. Moher D, Schulz KF, Altman DG for the CONSORT Group. The CONSORT Statement: revised recommendations for improving the quality of reports of parallel-group randomized trials. Ann Intern Med 2001;134 :657-62.

7. De Angelis D, Drazen JM, Frizelle FA, Haug C, Hoey J, Horton R, et al. Clinical trial registration: a statement form the International Committee of Medical Journal Editors. CMAJ 2004;171(6):606-7.

8. Easterbrook PJ. Directory of registries of clinical trials. Stat Med 1992;11:345-423.

9. Simes RJ. Publication bias: the case for an international registry of clinical trials. 7 Clin Oncol 1986;4:1529-41.

10. Dickersin K, Min YI. NIH clinical trials and publication bias. Online 7 Curr Clin Trials 1993; Apr 28(doc 50).

11. Dickersin K, Min YI, Meinert CL. Factors influencing publication of research results: follow-up of applications submitted to two institutional review boards.7AMA 1992;267:374-8.

12. Sterling TD. Publication decisions and their possible effects on inferences drawn from tests of significance — or vice versa. 7 Am Stat Assoc 1959;54:30-4.

13. Huston P, Moher D. Redundancy, disaggregation, and the integrity of medical research. Lancet 1997; 347:1024-6.

14. Chan AW, Hrobjartsson A, Haahr MT, Gøtzsche PC, Altman DG. Empirical evidence for selective reporting of outcomes in randomized trials: comparison of protocols to published articles. JAMA 2004;291(20):2457-65.

15. Chan AW, Krleža-Jerić K, Schmid I, Altman DG. Outcome reporting bias in randomized trials funded by the Canadian Institures of Health Research. CMAJ 2004;171(7):735-40.

16. MacLean CH, Morton SC, Ofman JJ, Roth E, Shekelle PG. How useful are unpublished data from the Food and Drug Administration in meta-analysis? 7 Clin Epidemiol 2003;56:44-51.

Correspondence to: David Moher, Clinical Epidemiology Group, Children's Hospital of Eastern Ontario Research Institute, 401 Smyth Rd., Rm. 210, Ottawa ON K1H 8L1; fax 613 738-4800;

dmoher@uottawa.ca

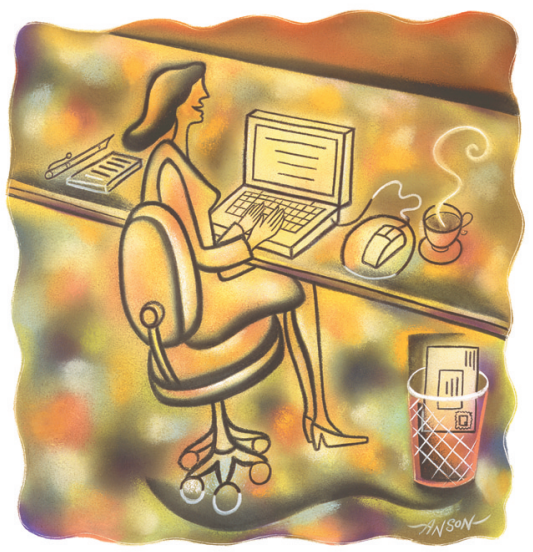

\section{Online manuscript submissions and peer review}

NOW AVAILABLE AT CMAJ http://mc.manuscriptcentral.com/cmaj 